\title{
Integrated optical coupling element for functional genomic analysis biosensor
}

\author{
Corinne Vergnenègre, Charlotte Bringer, Véronique Bardinal, Thierry Camps, Chantal Fontaine, \\ Antonio Muñoz-Yagüe \\ LAAS-CNRS, 7, avenue du Colonel Roche, 31077 Toulouse Cedex 4, France
}

\begin{abstract}
We present here a design of a coupling element aimed to be integrated into a nanomechanical biosensor for functional genomic analysis. The operation principle is based on a sub-nanometer resolution optical measurement of a cantilever deflection caused by a surface stress when the target nucleic acid sample hybridises to the nucleic acid probe on the active side of the cantilever. The resulting deflection, of the order of nanometers, is measured by an optical system, in which a laser beam reflects off the back of the cantilever to a position sensitive photo-detector. We study in this paper three polymer optical coupling systems which could allow to detect the presence of target nucleic acid on the cantilever by amplifying the deflection caused by the stress.
\end{abstract}

Key words : microsystem, optical deflection amplifier, nanometer optical detection, polymer

\section{INTRODUCTION}

The integration of optics in the Microelectromechanical systems (MOEMS), although it requires hybridisation, allows to achieve smaller, lighter, lower cost and more efficient systems compared to their macroscopic equivalent systems. Development of MOEMS has been emulated by the wide range of their potential applications in astronomy ${ }^{1}$, biology and medicine $^{2}$, industry ${ }^{3}$, or communication networks ${ }^{4}, \ldots$. These devices bring into operation integrated circuits with discrete elements (transistor, capacitors, resistors, lasers, ...) on a single chip and gather subsystems (electronics, mechanics, optics,...) on a multichip module, leading to increasing function panel and, as the same time, to decreasing volume, power consumption, and cost.

Recent progress in micro- and nanotechnology contribute to their development. In this article, we describe an optical sensor combined with microfluidic and active cantilever elements in an integrated DNA analysis. This sensor, composed of vertical surface emitting lasers (or VCSELs), an optical coupler, and a Position Spot Detectors (PSD), aims to detect and measure cantilever deflection due to DNA hybridisation. Laser beam is reflected off the cantilever to be tested, propagates in the coupling element and is detected by the PSD. The coupling element is used to amplify the laser beam deflection to be detected by the PSDs. Displacement detection of the order of the nanometer is expected to be reached without using resonant frequency technology, which implies the optical head has to be mechanically and thermally stable, and the deflection amplification gain important enough to generate a 0.1 ìm minimum displacement of the spot on the PSD.

The advantage of such an optical sensor lies in the use of VCSEL sources. These emitting devices can be fabricated as arrays whose pitch distance is consistent with that of cantilever arrays, rendering possible parallel detection.

Here, we will describe the design of the optical coupling element used to amplify beam deflection while ensuring system compactness. This study was carried out in order to identify what coupling element geometry could meet the microsystem requirements and optimise efficient beam deflection detection.

\section{SYSTEM SPECIFICATIONS}

Design of the optical sensor takes into account several constraints: size specifications, expected beam deflection on PSD, efficient light injection into (and collection from) the coupling element, as well as the control of the gaussian beam which tends to diverge along propagation. 
The beam size must be smaller than the width of the cantilever $(20 \mu \mathrm{m})$ in order to be efficiently reflected off its surface and kept below the pitch distance of the VCSELs (250ìm) along its pathway within the coupling element. The expected cantilever deflection lies in the 1-50nm range as the minimum displacement of the spot measured in terms of spot origin increment on the PSD is $0.1 \mathrm{im}$, the coupling element has to amplify the cantilever deflection in the ratio of $100-1000$ to achieve an efficient detection of the cantilever bending.

The laser sources are dielectric apertured VCSELs ${ }^{5}$ emitting at a wavelength of $850 \mathrm{~nm}$. The divergence of these monomode sources is smaller than of $10^{\circ}$ (FWHM). The optical coupling element would be made of polymer for fabrication convenience.

\section{FIRST OPTICAL COUPLING ELEMENT GEOMETRY}

\subsection{Description of the structure}

The first optical coupling module is shown schematically in

Figure 1. It presents a symmetrical geometry. The VCSEL source is located perpendicular to the microfluidic head and to the cantilever. This presents the advantage to allow for easy laser source and photodetector alignment relatively to the microfluidic module.

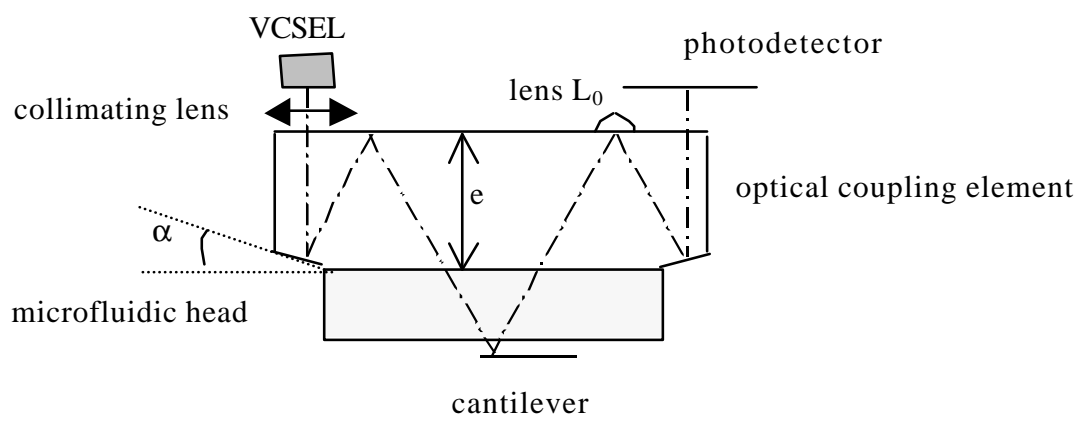

Figure 1 : Schematic view of the coupling module

The $\alpha$ angle has been chosen small $\left(5^{\circ}\right)$ to minimize the pathway between the source, the cantilever and the lens $L_{0}$. Indeed, the gaussian beam crosses the coupling element thrice before going through the microfluidic head and the cantilever.

A preliminary study showed we cannot prevent from using a collimating lens between the VCSEL source and the coupling element because of the source beam divergence and the beam size requirements. This collimating input lens has been designed in view of minimizing the gaussian beam divergence. It has been placed at the input extremity, and dimensioned with respect to the pitch of the system (250ìm). Moreover, it has been inserted on the coupling element surface in order to provide a compact integrated device.

The beam of the VCSEL source goes to the cantilever through the coupling element after being collimated. A second lens $\left(\mathrm{L}_{0}\right)$ has been added. This lens aims to increase the beam deflection due to the cantilever bending and thereby to improve PSD detection. As well known in the planar optics concept, the positioning of optical elements demands very high precision ${ }^{6}$. As a consequence, a special care has be brought to the design and the positioning of this lens. The output extremity has been tilted, with the same angle as for the input extremity, in order to set the device symmetrical and also to minimize the spot distortion on the PSD. 


\subsection{Performances and results}

The main difficulty is to optimise the spot deflection amplitude on the PSD while keeping a small waist spot on the detector. The gaussian waist increases as the beam propagates due to the beam divergence. For this reason, the pathway will have to be kept within a limit value in order to maintain the beam waist smaller than a few hundreds of micrometers. A short coupler geometry, allowing for a single to and through after the cantilever, has then been studied in order to evaluate the beam waist increases just after the lens $\mathrm{L}_{\mathrm{O}}$.

Taking into account these considerations, a first study turned to the thickness (e) optimisation of this coupling element. For a PSD placed $1 \mathrm{~mm}$ close to the coupler output face, we have studied the variation of the beam waist on the PSD as a function of e, the coupling element thickness, after one to and through and with the same lens $\mathrm{L}_{0}$ (see

Figure 1). The modelling showed an optimised thickness of the order of $1 \mathrm{~mm}$.

The second key parameter is the $\mathrm{I}_{\theta}$ lens characteristics. The following figures shows the variation of the spot displacement on the PSD and the beam waist at the coupler output when the radius of curvature of $\mathrm{L}_{0}$ varies (see Figure 2 and Figure 3).

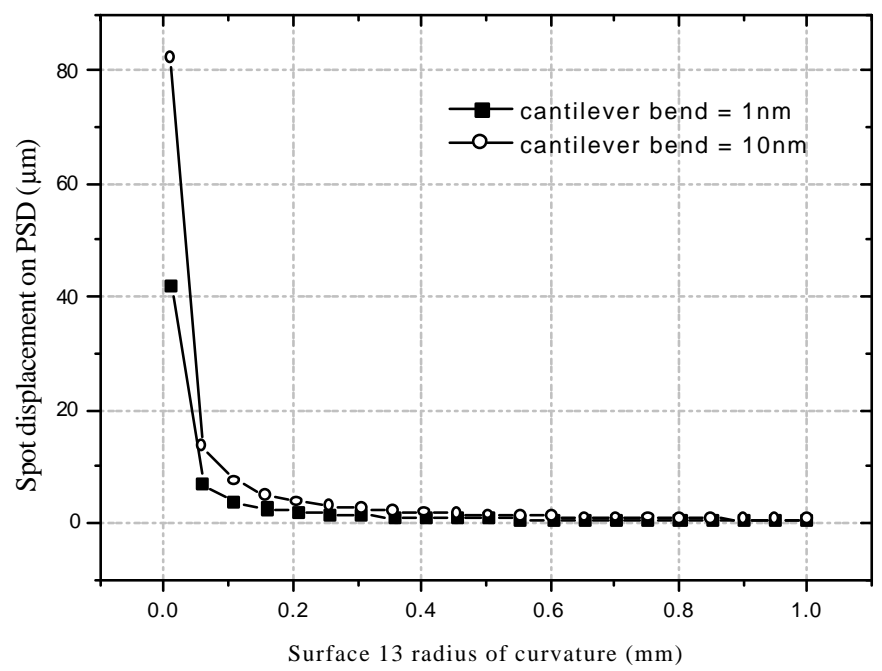

Figure 2: Displacement of the spot center on the PSD as a function of lens radius of curvature for a cantilever deflection of $0.02 \mathrm{~nm}$ and $0.1 \mathrm{~nm}$

These results show an exponential decay of the spot displacement when the lens radius increases. We can note that the spot displacement amplitude does not significantly evolve anymore as soon as the lens radius exceeds 0.15 to $0.2 \mathrm{~mm}$. To reach the accuracy of $0.1 \mathrm{~nm}$ (that is when cantilever bending generates a 0.1 ìm spot displacement on PSD), the radius curvature of the lens $\mathrm{L}_{0}$ must be equal to $0.056 \mathrm{~mm}$, as can be seen in Figure 2. Under these conditions, the device presents the following performances : the amplification coefficient, called a, which quantifies the spot shift amplitude on the PSD for a $1 \mathrm{~nm}$ cantilever bending is equal to $0.74 \mathrm{ìm} / \mathrm{nm}$. It means that a lìm spot displacement on the detector allows to detect a $0.135 \mathrm{~nm}$ cantilever deflection.

Meanwhile, the beam waist has to be controlled to provide the required device sensitivity and avoid cross talking between adjacent ways. The variation of the waist as a function of the lens radius beam is shown in Figure 3 and shows that a lens $\mathrm{L}_{0}$ with a large radius of curvature has to be used. Unfortunately this is opposite to the conclusion drawn concerning the beam displacement optimisation. Indeed, the radius previously proposed to meet the latter requirement was $0.056 \mathrm{~mm}$, which would lead to a $3.9 \mathrm{~mm}$ spot on the PSD. 


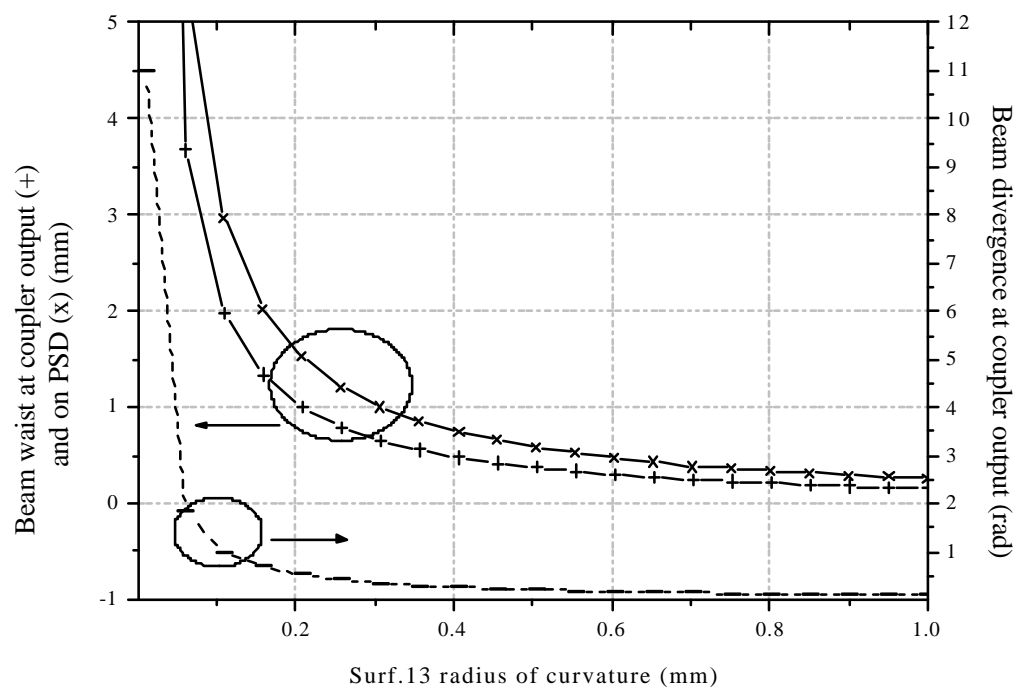

Figure 3 : Evolution of beam waist as a function of radius of curvature of lens

\subsection{Conclusion}

This study shows that this geometry, in spite of its advantage for system alignment, is not suitable for the aimed application. This architecture is potentially efficient in terms of spot displacement on the detector : the radius of curvature of the lens $\mathrm{L}_{0}$ is a useful parameter which allows to easily choice the performances of the coupling element. The study shows the smaller the radius is, the greater the spot deflection is. Notwithstanding, a small radius is unfavorable to the beam waist on the PSD.

\section{SECOND OPTICAL COUPLING ELEMENT GEOMETRY}

\subsection{Description of the device}

The design of the first coupler has been mo dified in order to keep the deflection spot amplitude we have reached in the precedent part, while reducing the beam spot on the PSD. To manage this trade off, the VCSEL source has been tilted with respect to the cantilever, and the lens $\mathrm{L}_{0}$ placed after the beam reflection by the cantilever has been maintained, as can be seen on Figure 4. Indeed the path way between the VCSEL source and the cantilever is divided by a factor 3 compared to the precedent design, which prevents the gaussian beam from diverging along the part of optical path which don't contribute to the amplification deflection. We have kept the same integrated collimating lens as in the precedent study, as it decreases the beam divergence to less than $1^{\circ}$ in the coupling device.

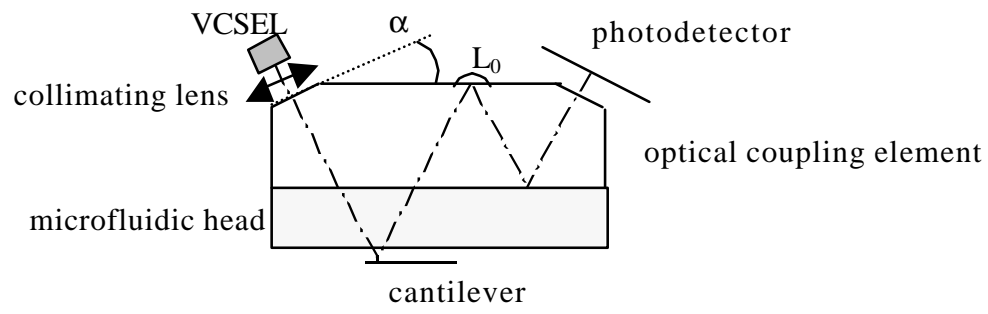

Figure 4 : Side view of the second coupler 


\subsection{Performances and results}

In this configuration, the a coefficient which quantifies the deflection gain of the coupling element is equal to $0.656 \mathrm{im} / \mathrm{nm}$. The deflection performance remains stable (compared to $0.74 \mathrm{im} / \mathrm{nm}$ ), while the gaussian beam waist decreases from $3.9 \mathrm{~mm}$ to 831 ìm on the PSD.

We can conclude that this design partly improves the gaussian beam waist on the detector, but its performances are not sufficient enough to meet the system requirements.

\section{THIRD OPTICAL COUPLING ELEMENT GEOMETRY}

\subsection{Description of the device}

Taking into account these conclusions we have study a coupling element geometry which would allow us to keep a small divergent beam and to amplify the cantilever deflection in the same time by lengthening. A side view of the coupling element is shown in Figure 5. The beam reflects off the back side of the cantilever and then is guided via the coupling element to the output face and the PSD. As for the second geometry, the VCSEL source and the photo-detector are tilted with respect to the cantilever. We have kept the symmetrical structure (input and output faces) to avoid spot distortion on the PSD.

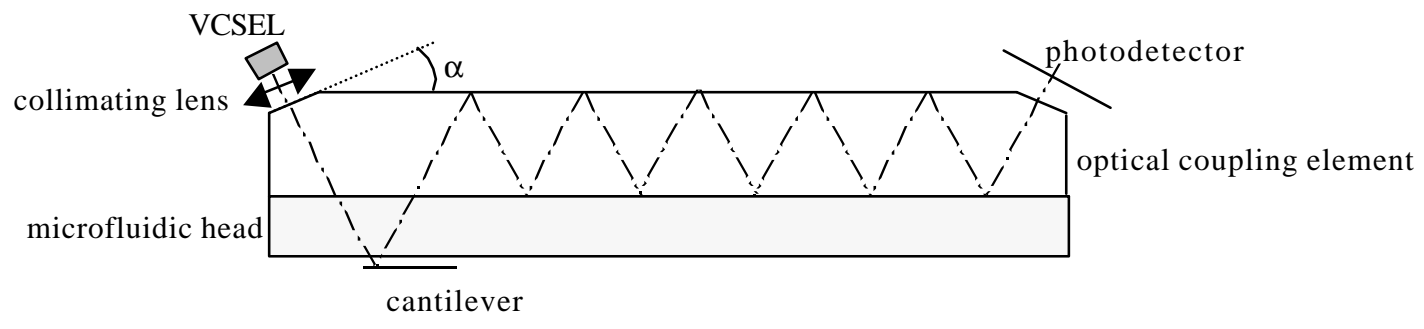

Figure 5 : Side view of the third coupler

The beam is still collimated by means of a collimating lens as in the other designs. This decreases the beam divergence from $10^{\circ}$ (FWHM) at the source point to $0.94^{\circ}$ inside the coupling element. The thickness of the coupler and the $\alpha$ incident angle have been kept equal to $1 \mathrm{~mm}$ and $20^{\circ}$ respectively.

\subsection{Performances and results}

The spot displacement on the PSD has been evaluated after 6 to and through in the coupling element, which corresponds to a path length of $4.75 \mathrm{~mm}$ between the cantilever and the PSD (see Figure 6). This figure shows a relative low gain as the gain coefficient is $0.092 \mathrm{im} / \mathrm{nm}$. This confirms the need to lengthen the optical path in the coupling element in order to reach the required accuracy. However it is worth noting that this design leads to a propagating beam of good quality: the spot waist of the gaussian beam on the cantilever and the PSD are respectively 18ìm and 51.5ìm.

To emphasize the amplification gain, longer couplers have been studied. Results are shown in Figure 7 where $\mathrm{L}$ is the total optical path length covered by the light between the cantilever and the PSD, and a is the deflection gain factor.

From this figure, we have inferred the performances of the coupling element (that is the expected spot displacement on the PSD) as a function of the optical path length, for a $1 \mathrm{~nm}$ cantilever deflection. The results are shown in Figure 8 . They show that the ratio $\Delta \mathrm{z}(\mathrm{nm}) / \mathrm{L}(\mathrm{mm})$ is around $36 \mathrm{~nm} / \mathrm{mm}$. 


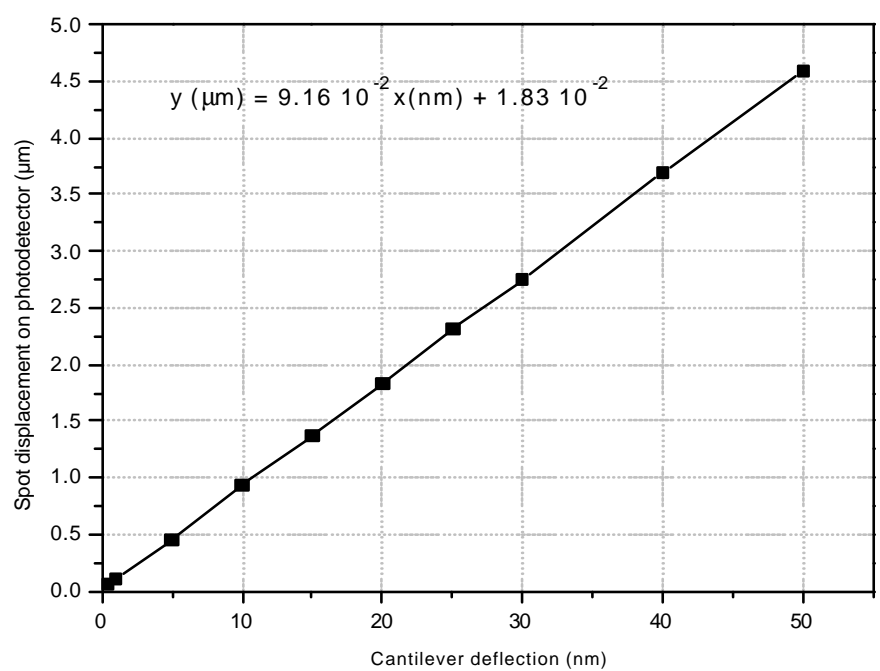

Figure 6 :Spot displacement on the PSD for 6 to and through in the coupling element

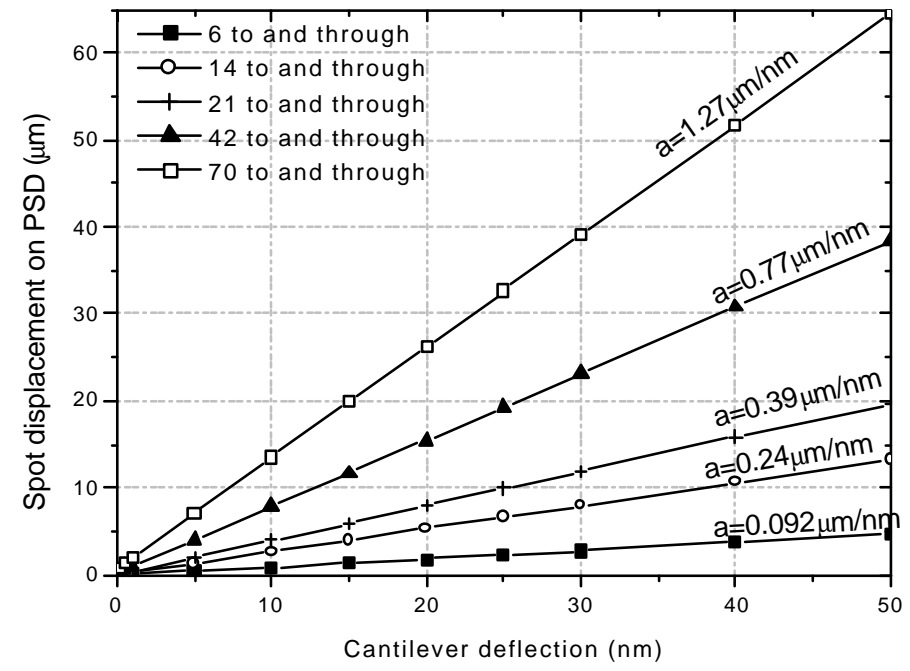

Figure 7 : Spot displacement on PSD for different coupler lengths

The complete results are summarized in Table 1 . The a coefficient is directly related to the resolution of the device : to the extent that the minimum spot origin displacement on the PSD is 0.1ìm, the minimum cantilever deflection which can be detected by the PSD can be calculated. This resolution decreases as the path length in the optical coupling element increases. 


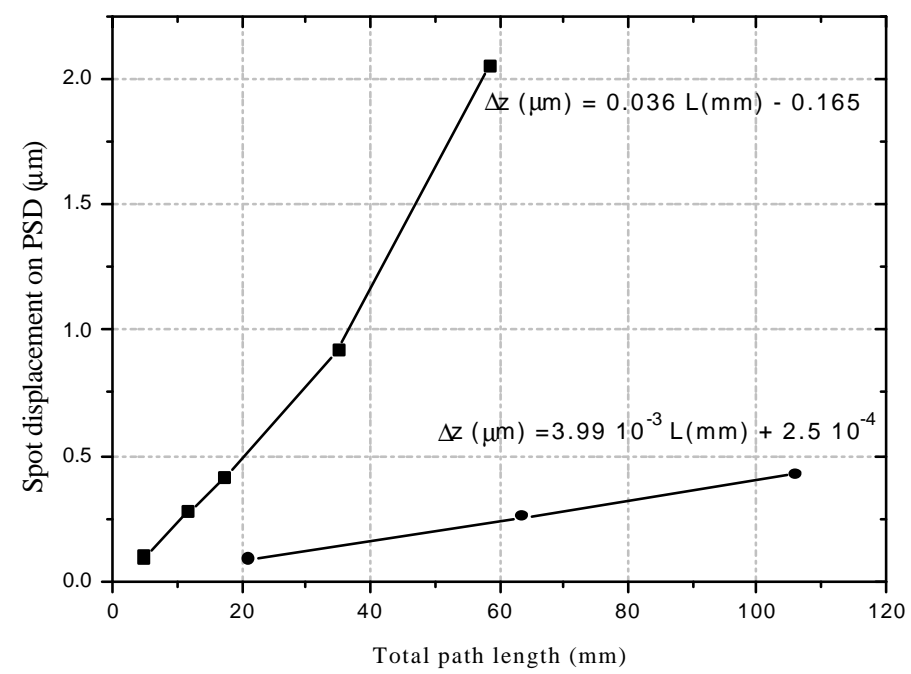

Figure 8 : Spot displacement on the PSD as a function of path length for a $1 \mathrm{~nm}$ cantilever bend

\begin{tabular}{|c|c|c|c|}
\hline $\begin{array}{c}\text { To and through } \\
\text { number }\end{array}$ & $\begin{array}{c}\text { Cantilever-PSD } \\
\text { total pathway } \\
(\mathbf{m m})\end{array}$ & $\begin{array}{c}\text { Spot diameter } \\
\text { on PSD (im) }\end{array}$ & $\begin{array}{c}\text { Resolution } \\
\text { (for 0.1ìm spot } \\
\text { displacement on PSD) }\end{array}$ \\
\hline 6 & 4.75 & 51.5 & $1.087 \mathrm{~nm}$ \\
\hline 14 & 11.6 & 117.5 & 0.380 \\
\hline 21 & 17.4 & 170.6 & 0.253 \\
\hline 42 & 34.9 & 327.7 & 0.130 \\
\hline 70 & 58.5 & 548.1 & 0.078 \\
\hline
\end{tabular}

Table 1 : Results of the coupling element for different to and through numbers

From these results, we can also estimate the coupling element length required to achieve the $0.1 \mathrm{~nm}$ required accuracy. To reach this goal, the variation of the resolution as a function of the total pathway in the coupling element has been drawn in Figure 9. We can observe that the resolution exponentially decreases with the optical pathway in the coupling element. This implies a minimum length of $45 \mathrm{~mm}$ to reach the $0.1 \mathrm{~nm}$ resolution.

Until now, only geometrical considerations have been taken into account. Nevertheless, the fact that the source is a gaussian beam must be kept in mind. As the path length increases, the gaussian spot diameter increases too, according to the well known variation law of the gaussian beam waist. The variation of the spot waist on the PSD as a function of the path length is also shown in Figure 9.

So, according to the allowed spot diameter on the PSD, it appears that the coupler length will be limited, and consequently the lower detectable cantilever bending. In any case, the tolerable spot diameter should not overstep the VCSEL pitch, i.e. 250ìm. As an example, in the case where the aimed spot diameter equals 100ìm, the maximum permitted path length is equal to $10 \mathrm{~mm}$ and the corresponding resolution is around $0.45 \mathrm{~nm}$. 


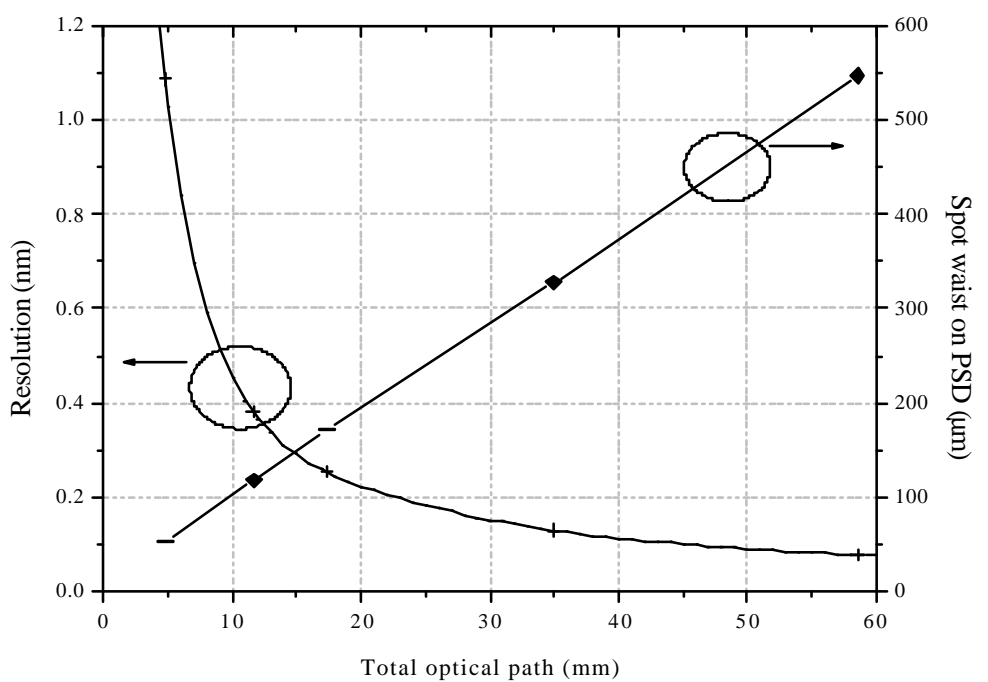

Figure 9: Variation of the resolution as a function of the total optical path

\subsection{Conclusion}

As a conclusion of this part, we have shown that we can reach the required resolution by lengthening the optical coupling element. The minimum length needed to achieve the $0.1 \mathrm{~nm}$ resolution is equal to $45 \mathrm{~mm}$. For this resolution, the spot waist on the PSD is equal to 423ìm.

\section{SUMMARY}

We have proposed three architectures for the optic al coupling element to be used as a part of a biosensor microsystem. In the first one, the control of the beam waist is too critical to provide a stable and reliable element suitable for the aimed application. This can be improved by slightly changing the geometry but not sufficiently. Nonetheless, the last proposed coupling element is based on the propagation of a very few divergent beam. This solution presents the advantage of an important stability as far as the beam spot diameter is concerned, and allows to adjust the required resolution of the system by selecting the right device length.

\section{ACKNOWLEDGMENTS}

This work is granted by the EU contract IST-2001-37239. We would like to thank the project partners participating to this proposal : CRL, Ltd (UK), CNB-CSIC and CNM-CSIC (Spain), and Genetrix, SL (Spain).

The simulations have been computed thanks to the Synopsys lens design program (Optical System Design Inc.).

\section{REFERENCES}

1. F.Zamkotsian, K.Dohlen, D.Burgarella, M.Ferrari, V.Buat, MOEMS, Key Optical Components for Future Astronomical Instrumentation in Space, ICSO 2000, $4^{\text {th }}$ International Conference on Space Optics, Toulouse, France, 5-7 December 2000.

2. J.Tamayo and al., Integrated Prototype of Nanomechanical Biosensor based on Silicon Technology for Biosensor Applications, to be published. 
3. J.R.Wendt and al., Fabrication of optical elements for an integrated compact optical microelectromechanical system laser scanner, J. Vac. Sci. Technol., B 18 (6), Nov-Dec. 2000.

4. R.Houbertz, M.Popall, ORMOCER®s for Micro-Optical and Integrated-Optical Applications, Data extracted from the Fraunhofer ISC Annual Report 2001.

5. V.Bardinal, L.Averseng, C.Bringer, T.Camps, J.Polesel-Maris, P.Dubreuil, C.Fontaine, E.Bedel-Pereira, C.Vergnenègre, A.Muñoz-Yagüe, Experimental demonstration of oxide modes influence in a dual-purpose oxideconfined vertical cavity surface emitting laser, Applied Physics Letters, Vol.81, nº10, pp.1771-1772, Sept.2002.

6. Micro-Optics, Elements, systems and applications, Edited by Hans Peter Herzig, 1997, 359p. 